\title{
The effect of representation location on interaction in a tangible learning environment
}

\author{
Sara Price ${ }^{1}$, Taciana Pontual Falcão ${ }^{1}$, Jennifer G. Sheridan ${ }^{1}$ \& George Roussos ${ }^{2}$ \\ ${ }^{1}$ Institute of Education \\ London Knowledge Lab \\ 23-29 Emerald Street, \\ London, UK \\ WC1N 3QS \\ s.price, t.pontual,j.sheridan $\{@$ ioe.ac.uk $\}$ \\ ${ }^{2}$ Department of Computer Science, \\ Birkbeck College, \\ London Knowledge Lab \\ 23-29 Emerald Street, \\ London, UK WC1N 3QS \\ g.roussos@dcs.bbk.ac.uk
}

\begin{abstract}
Drawing on the 'representation' TUI framework [21], this paper reports a study that investigated the concept of 'representation location' and its effect on interaction and learning. A reacTIVision-based tangible interface was designed and developed to support children learning about the behaviour of light. Children aged eleven years worked with the environment in groups of three. Findings suggest that different representation locations lend themselves to different levels of abstraction and engender different forms and levels of activity, particularly with respect to speed of dynamics and differences in group awareness. Furthermore, the studies illustrated interaction effects according to different physical correspondence metaphors used, particularly with respect to combining familiar physical objects with digital-based table-top representation. The implications of these findings for learning are discussed.
\end{abstract}

\section{Keywords}

Tangible interface, representation, children, learning.

\section{INTRODUCTION}

Recent reviews and literature searches on the value of tangibles for learning have shown a paucity of research that specifies the relationship between tangible interaction and learning benefits $[14 ; 16 ; 21]$. A research framework has recently been developed for investigating the specific benefits and disadvantages of the various design parameters of tangible environments in the context of learning [22]. This framework places emphasis on the central role of external representation (both digital and physical) in tangible learning environments, and is structured around the different physical-digital representation relationships that occur in tangible environments. A focus on representation offers the means to better understand the role of tangibles for learning, specifically as learners are required to make meaningful mappings between both artefacts and action.

The framework includes both artefact-representation LEAVE BLANK THE LAST $2.5 \mathrm{~cm}$ (1 ") OF THE LEFT COLUMN ON THE FIRST PAGE FOR THE COPYRIGHT NOTICE. relationships and action-representation relationships, which form the basis for exploring the learning implications of different design choices. The framework has four primary parameters: The location parameter refers to the different spatial locations of digital representations in relation to the object or action triggering the effect; (ii) dynamics is concerned with the flow of information during interaction, including how information is linked between action, intention and feedback; (iii) correspondence refers to the metaphors involved in the nature of representations of artefacts and actions placed upon them. The modality of representation impacts on different aspects of the whole interaction and can be considered in parallel to all other categories [22].

The study and findings presented in this paper centre around particular aspects of two parameters: location and correspondence. The instances discussed in this paper are highlighted in thick black edges in Figure 1, which depicts the complete framework.

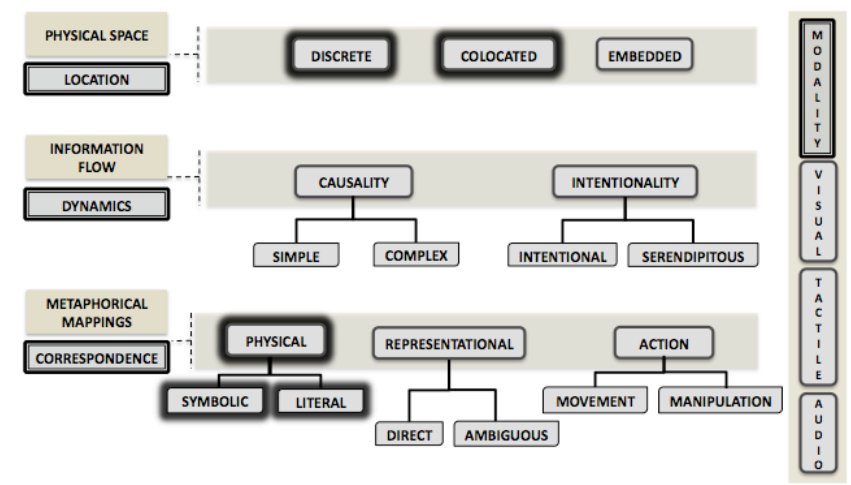

Figure 1. The representation framework, with instances of the location and correspondence parameters highlighted.

\section{Location}

The location parameter identifies the different possible locations of digital representations in relation to the object behaving as input device, or the action performed upon it, in physical space. Three categories are identified:

- Discrete: refers to a representation, which is located separately from the input device (or tangible object). For example, in Smartstep [26], the result of children's physical action (jumping) on a sensitive mat is shown on 
a computer screen, which mirrors the mat and augments it with relevant digital information.

- Co-located: occurs when input and output are contiguous, and the digital effect is directly adjacent to the artefact or action. For example, in the Illuminating Light system [29], users move physical representations of optical elements on a tabletop, and the system projects on the same surface the correspondent propagation of laser light.

- Embedded: is when the digital effect occurs within the object e.g. an object lights up, changes colour or vibrates. For example, the Flowblocks [30] are concrete blocks that send light signals through when connected together.

As learners are required to make meaningful links between objects, action and effects, these location couplings may affect various aspects of cognition, for example, explicitness of artefact-representation links and subsequent attention demands may differ between discrete and colocated coupling.

\section{Correspondence}

The correspondence parameter identifies the different meaning (metaphorical) mappings that occur between object or action and representation. Mappings might relate to the physical properties of the object, the representational mappings or action mappings. Price et al [22] suggest that "investigating how these mappings facilitate understanding of scientific concepts, or engender effective learning strategies (e.g. cognitive conflict, reflection, exploration) is essential for understanding how such correspondences can best support learning" [p.360]. Physical correspondence refers to the degree to which the physical properties of the objects are mapped to the learning concepts, the emphasis being on the degree of correspondence to the metaphor of the learning domain, and how this constrains or influences inferences and conceptual understanding. There are two categories within the physical correspondence parameter:

- Symbolic: which defines objects that act as common signifiers, and which may have little or no characteristics of the entity it represents.

- Literal: which defines objects whose physical properties closely map onto the metaphor of the domain it is representing.

The research reported here focuses on (i) the location parameter, in particular exploring the differential costs and benefits of discrete and co-located representations; and (ii) the correspondence parameter, in particular the concepts of physical correspondence. This study explores their impact on interaction in tangible learning environments.

\section{BACKGROUND}

A number of frameworks and taxonomies for conceptualising tangible user interfaces provide different ways of classifying tangible interfaces to describe, compare or analyse the different systems. Some focus on the technical aspects of the system [e.g. 28], others provide descriptive analyses [2], while yet others have begun to outline interactive features of tangible environments that provide a basis for analysing interactions and their effects $[6 ; 10]$. However, few frameworks attempt to define tangible interfaces from the perspective of learning.

Research has extensively demonstrated the important role of external representations in mediating cognition. Although, this is largely based on visual representation research [e.g. 24;12;1;20], it has also been demonstrated with other modalities, such as audio [e.g. 4]. Much of this work focuses on the particular design of the external representations themselves, and the cognitive interaction that they engender. However, with tangible environments the cognitive relationship with external representations becomes increasingly complex, as it not only depends on representation design, but also the links between the physical world or artefacts and related representations.

One factor for tangible environments and interactive displays concerns the location of input and output in terms of representation effect on interaction. Different physical orientation of displays (vertical versus horizontal) has been shown to have an impact on collaborative group working [23]. Horizontal surfaces were found to promote more fluid collaborative interaction through close coupling of physical and digital information and increased affordance for role swapping, than the vertical surface. However, this comparison used mouse-based interaction where control of the environment was constrained, and sharing or switching control was awkward. In the tangible environment presented here input occurred through several networked objects, which can be used simultaneously by all users, precluding the need to 'share' or 'pass on' control. The same objects were used in both the co-located (horizontal display) and discrete (vertical display) conditions.

A further study exploring different representational location designs in a tangible application [17] showed that users preferred information displayed on the sensing surface (horizontal) rather than on a separate screen (vertical), as this meant that they did not have to divide their attention between the input (sensing surface) and the output (separate screen display). This study seeks to go beyond user preference to understand the interactive and cognitive effect of the different designs.

Another area of related research explores the effect of different input and output on awareness levels of users in table-top environments [15; 18]. Pinelle et al. [18] showed that direct touch generated higher levels of awareness of others location or activity, but that virtual embodiments through relative input were easier to track the people's movement through touch. Although a different approach, some features of direct touch and virtual embodiment through relative input techniques (such as the mouse), can be related to concepts of co-located and discrete representations respectively, particularly with respect to awareness levels.

A second factor concerns the mappings that are required between artefact or action and digital effect. Tangible 
environments can rely on direct mappings between physical action and representations on computer screens [e.g. 25; 26], which enable concrete experience with abstract concepts. Despite the common belief that abstract concepts are more easily grasped through experience with concrete representations, connections between physical objects and underlying abstract concepts are not always transparent to students. Furthermore, concrete representations may limit students' comprehension to a specific context, and prevent access to the general nature of the abstract concept [5].

\section{THE TANGIBLE ENVIRONMENT}

\section{Table-top design}

A purpose built tangible environment was developed to support students learning about the behaviour of light. Specific concepts explored include reflection, transmission, absorption and refraction of light, and derived concepts of colour. The tabletop environment (Figure 2) was custom made and draws from the design of the reacTable, which employs reacTIVision technology for object recognition [8; 9]. Interaction was enabled using a variety of custom-made artefacts tagged with fiducial icons. Applications are developed using the Processing language. Multiple objects can be recognized simultaneously thus enabling several participants to interact with the tabletop together. Users interacted with the application using a set of different coloured acrylic blocks and a torch, which were tagged with fiducial markers. Interaction consisted of placing and moving the tagged blocks and torch on the table surface.

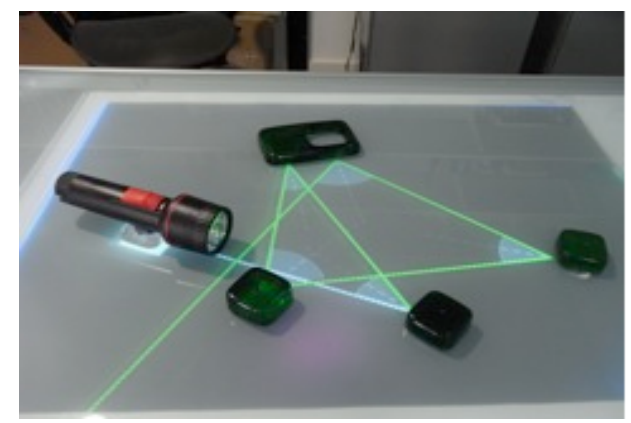

Figure 2. The tabletop environment in co-located mode running an application on the Physics of light

Visual effects, projected on the table surface showing light reflection, absorption, transmission and refraction, were triggered when users manipulated the torch and the blocks on the table surface. All of the objects simulated real-world behaviours, i.e., the torch shone light and the blocks reflected, absorbed and / or transmitted light according to their colour and opaqueness. For instance, according to the Physics of light a block looks green because it reflects green light. In our application pointing the torch at a green block caused a green beam to be reflected off the block (Figure 2).

The blocks and torch must be on the table surface for the system to recognize them as interaction elements, and for the corresponding digital effects to be displayed. The digital effects can appear either on the table surface, adjacent to the objects (following the co-located approach presented in Figure 2) and / or projected onto a separate vertical display such as a wall (according to the discrete approach presented in Figure 3). In the discrete approach, digital representations of the physical objects are shown on screen as well as their corresponding digital effects. The embedded approach was not explored in this particular iteration of the tabletop environment.

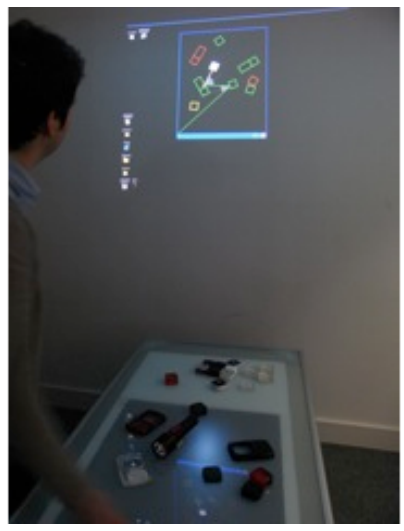

Figure 3. Tabletop running in the discrete mode Representation design

During design and development of the discrete versus colocated versus embedded representation, it became apparent that each location of representation lends itself to different kinds of representation, that can be described in terms of levels of abstraction. For example, an embedded representation lends itself to refer to something going on inside the object, as in the case of Flowblocks [30]. In the case of the 'light' concept, representations embedded in the object itself would have to show something related to 'inside' the object, such as the light waves being absorbed, or transmitted. When designing the discrete and co-located representations it became evident that some concepts can more clearly be illustrated on the discrete that the colocated. For example, showing the way the light beam refracts when transmitted through an object is problematic on the co-located system, as the object itself is 'covering' the surface where the digital image is displayed. Whereas on the discrete representation, the object is located on the table surface and the digital representation, displayed on a separate screen, can easily show the pathway of a beam as it travels through the object (Figure 4).

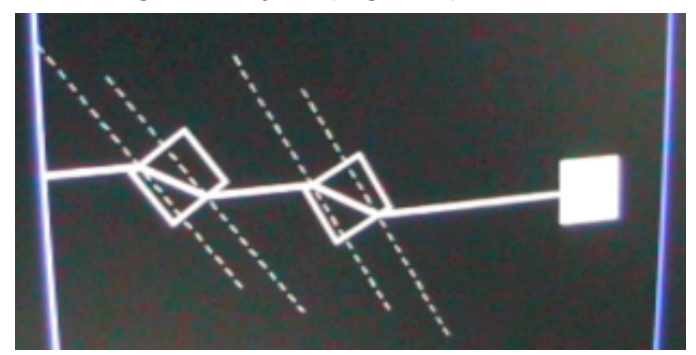

Figure 4. Refraction inside objects shown on screen

These design findings suggest that by combining different representation locations in one system offers opportunities for illustrating phenomena at different levels of abstraction. 
This is compelling particularly within science where complex phenomena often need to be understood at different levels of abstraction.

\section{STUDY}

An initial study was undertaken to evaluate the tangible environment in terms of usability, engagement, collaboration, and conceptual inferences arising from the interaction and locations and metaphorical mappings [22]. In this paper, we focus on the findings relating to two aspects of the representation-based framework [22]; location and physical correspondence and their influence on interaction and meaning-making.

\section{Study design}

The study involved 21 children aged 11 years (11 female and 10 male), from two schools in the outskirts of London (UK). Children worked with the tangible system in groups of three, consisting of a mixture of girls and boys, making seven groups of children. A reverse order repeated measures design was used, with three groups assigned to co-located followed by discrete; and 4 groups assigned to discrete followed by co-located. This design enabled children's comparative assessment as well as the researchers. The study was explicitly designed to investigate the two locations mentioned, while the aspects regarding correspondence were implicit in the choice of the interaction objects and design of the application (i.e. using a real torch and blocks that behaved as in the real world). The effect of these choices regarding the correspondence parameter was observed and analysed throughout the interaction with the two locations.

\section{Study procedure}

As the aim of the study was to investigate the effect of tangible interaction on children's reflection and meaningmaking, rather than learning outcomes, no pre-tests on content were administered, but informal conversations with each group elicited their ideas about light behaviour and introduced the context of the activity. Some children were aware of basic 'light' concepts such as light traveling in straight lines, shadows, and opaque and transparent objects.

Each session lasted 35-45 minutes. Children were asked to freely explore the interface (by moving the objects on the tabletop) to find out about light behaviour. During the interaction, the facilitator prompted the group with general questions like "what's happening here?" and "why do you think this is happening?" to guide students through the exploration of the concepts towards making inferences and drawing conclusions. All sessions were video-recorded. After engaging with the tangible system children were interviewed in their groups to obtain information on their understanding of key concepts of light behaviour, feedback on representation location and the system as a whole, and their general experience.

Collected data (video-recordings and post experience interviews) were qualitatively analysed, focusing on the emerging aspects that related to the impact of the two locations and emergent findings related to concepts of correspondence.

\section{FINDINGS AND DISCUSSION}

All of the children found the environment easy to use and found interaction with the objects and table unproblematic. Overall, every student liked the environment. Although they found this hard to articulate, their perception of enjoyment was directly related to their perceived level of activity, stating for example, "because you're actually doing it"; or "you would remember what you've been doing because it was fun".

Although the role of the tangible environment in supporting learning processes and in situ engagement with learning concepts is discussed in detail elsewhere [19], evidence from their interaction, and explanations with demonstration to their teachers after the activity suggest that children were grasping the concepts. For example, the majority of the groups commented on and questioned the fact that reflection from 'rough' objects did not generate subsequent reflection from other objects of the same colour, claiming that it should. They were in fact correct, as this instance of software implementation was incomplete.

\section{Location}

This study was primarily concerned with comparing the two location conditions. Thus consistency of representation across conditions was maintained where possible, rather than introducing the levels of abstraction discussed earlier. Thus, the primary illustrative difference between the two locations was the explicit representation of the change in beam direction during transmission in the discrete condition. However, children in the study did not easily notice this change in beam direction and continued to infer that the beam was 'going straight through, still'. After some persistence the facilitator got some pupils to notice refraction on the wall, but in spite of this, the postinterviews showed that children were not aware of the beam refraction when transmitted through an object. This may be due to a number of reasons. First of all, refraction does not occur when the incident beam hits the surface at a right angle, therefore light could indeed go straight through, without bending, depending on the orientation of the object involved. Second, due to technical limitations, the projected image was not very large and could become confusing when many objects were represented. In this case, pupils would not easily link each individual object to its virtual counterpart (especially when identical blocks were shown and position was the only variable to tell one from the other) and aspects related to one specific object could go unnoticed. Furthermore, children did not easily see digital representations as the objects' virtual embodiment: when prompted about was going on 'inside' the objects, pupils commonly came closer to look through the concrete block itself, rather than using the projected images of the objects.

\section{Preference}

Children expressed mixed opinions between the discrete and co-located arrangements, although the vast majority 
preferred the co-located. This is coherent with previous research that shows adult students preferences for output on a sensing surface rather than vertical display [17].

When discussing the difference between the two, one group claimed that they found the discrete mode confusing. Another group liked the table best "because you can learn what you are doing", and "you don't really have to ask any questions because you find out the questions about what you are doing yourself". One child equated the screen with an interactive whiteboard and claimed the table was easier because you could touch it and work with it. It could be argued, though, that strictly speaking those reasons do not relate solely to one location - as in the discrete mode objects are also manipulated and you can 'do it yourself', only the coupled effects are shown elsewhere. The colocated approach may, however, convey more the impression of concrete and active interaction.

Another claim made was that "you can see properly what's going on, you can see what you're doing and you don't have to keep looking up there". During the post interviews, one child began to explain why the screen was better, but ended up giving a reason why it was not! i.e. "you have to keep looking up and down all the time". One possible explanation might be that following interaction with mouse and keyboard it is fairly intuitive to look at the screen while your hands are doing something else, so children assume that this is more 'normal', but discover through articulation that in fact the table-top display removes this problem and feedback is more directly viewed.

One group, however, displayed a lot of excited exclamations with the screen display, and was actively engaged in moving around the blocks and the torch to create different light effects. This group expressed preference for using the discrete design, and one girl claimed that it was easier to find out what colours the blocks were on the screen. This may have been because the physical object colour was sometimes harder to distinguish in the darkened room, whereas the visual screen representation of the block was akin in clarity to the reflected light beams on the table.

In interview one child stated that moving objects with the display on screen (i.e. discrete) was a slower process, and therefore gave more time to think about what was happening. This is interesting particularly in relation to analysis of levels and type of activity (next section).

\section{Activity}

From the analysis particular aspects of interaction and levels of activity were found to differ between the colocated and discrete modes. In the discrete version, interaction with the system did not solely depend on the torch (as in the co-located), because each block on the table had a corresponding visual representation on the screen display. Although light behaviour effects only happened with the torch, this feature of the discrete mode meant that each child could play on their own with the blocks and their representations. For example, one boy did not get involved in the discussions, but played on his own, building several arrangements with blocks on the table and observing the virtual patterns made on the wall. This would not be of interest in the co-located mode, as building arrangements in this mode does not involve the digital visual effects, as the block is representing itself.

In contrast the co-located version required the torch (or light source) in order to elicit digital effects with the blocks. As there were several blocks but only one light source, there were often sequences of rapid changes e.g. different coloured block reflecting different coloured beams as each child experimented with a different block. Although this meant that each one interrupted another's arrangement, it also provoked children to question what was happening. For instance, as one girl placed a block on a coloured beam, reasonably expecting to see it either 'blocked' or reflected, another child moved the torch, completely changing the whole arrangement and bringing great surprise to the girl, who at first thought her action had caused the changes. This simultaneous interaction exposed the pupils to more exemplary instances of light behaviour.

In general, this rapid dynamic interaction resulted in a 'buzzing' activity level with the co-located representation, with all children in the group interacting with objects straight away. This was also obvious when moving from the discrete to the co-located, as there was a clear contrast between their engagement, and the immediate effect of the co-located version. Children tended to suddenly start moving lots of the objects to elicit effects, together with increased verbal exclamation and expression. One child exclaimed "ahh you can see" - this emphasis suggesting that the illustration of concepts is clearer with the adjacent representation. In contrast when moving from co-located to discrete, one girl exclaimed "wow, that's hard, looking up there and moving it!"

With the discrete version children tended to be quieter, engaging in less verbal and movement-based interaction. In addition their actions, or the changes made by moving the objects, were slower and less frequent. For example, during initial exploration one group of girls slowly and silently put all objects together in front of the torch. They were very quiet and cautious of interfering with each other's actions. Other groups, although moving objects on the table, also exhibited silent interaction.

Two key implications for interaction and learning arise from these differences: one relates to concepts of awareness of others' interaction in co-located environments (where users are physically together); the other relates to questions around the dynamic speed of seeing changes with colocated representations versus slower interaction and reflection with discrete representations.

Concepts of awareness: In the co-located approach, users easily perceive other's body movements, which makes them (naturally) aware of who is doing what and where [18]. Bodily clues allow users to anticipate other's actions and leads to better implicit coordination of interaction, 
where verbal communication many times is unnecessary [8]. As for the discrete approach, as attention is directed to a separate screen, information about peers' actions is encoded in their 'virtual embodiment' [18] - which would be, in the case of this study, the object that the user is manipulating. However, users freely grab, drag and drop different objects, i.e. constantly change their virtual embodiment. Awareness of other's actions becomes a hard task in this context, and impedes interpretation of 'interference' events. In the co-located mode, interference in peers' actions and dispute of resources happened more often. The higher level of awareness and visibility within the group provided children with the possibility of explicitly preventing peers from performing actions that would clearly interfere in their own arrangements by taking objects from other's hands, but also supported them in working together towards a common goal. Furthermore, it offered clearer opportunities for linking actions of others to interference events, facilitating interpretation of effects. It is important to note that 'interference' need not be negative, but can serve as a productive form of collaboration.

Speed of changes: Several children simultaneously moving different objects creating different coloured reflections, often interrupted another's arrangement. Although this may be eased by introducing more light sources, any movement of torches would also result in multiple effects. The rapidity of the dynamic changes and the issue of linking multiple changes to one's own or anothers' action, may make it harder for students to think about the concept, due to the transient nature of the representations, and the frequent lack of a permanent or even semi-permanent 'residual' of one action or arrangement. Thus, in the co-located version the dynamic nature of the environment made it difficult for children to pursue different thoughts or ideas at the same time. Indeed, observation of one group of quiet girls, who moved few objects at a time, showed changes in the environment were made slowly. Thus, arrangements were often kept static for some time, allowing discussion, and reflection, to take place. These findings are coherent with other research on dynamic representations (e.g. animated diagrams), which suggest the transient nature of explicit depiction of dynamics [27], and simultaneous multiple information representation [13] increases memory load and processing demands [20].

However, it was apparent from our studies that it was precisely this dynamic nature of the environment and the facility to simultaneously explore concepts which interrupted or changed others arrangements, that promoted engagement with the activity and engagement with the concept. The rapid updating and changes in light behaviour due to movements of objects around the light source provided more instances and different examples of the behaviour of light from which children could draw conclusions. This is a central feature for supporting exploratory and experiential learning. Furthermore, the design of the environment makes it is relatively easy to recreate and therefore re-access ideas later, and repetition or slight variations of 'light behaviour' set-ups can easily be achieved. In the discrete mode, although the dynamic nature of the environment is essentially the same, the extra cognitive effort to link action on the table's surface to onscreen effects slows interaction down. However, that does not mean children have more opportunity to reflect on the scientific concepts, as they are struggling with issues on awareness and motor coordination, leading also to lower levels of group collaboration.

\section{Physical correspondence}

\section{Correspondence and implications for interaction}

In terms of physical correspondence there was evidence that constraints of the interface had effects on interaction. For example, using the reacTIVision technology requires all objects to be tagged with fiducials, which need to be placed directly on the table for the system to function. Picking up the objects means that they are no longer recognised by the system. This generates some key design issues - namely, using the object as you would in 3D space rather than on a $2 \mathrm{D}$ surface. This is particularly noticeable with the torch, which worked through fiducial tagging rather than batteries.

All groups began by trying to use the torch in a familiar way. This involved switching it on, picking it up and moving it around freely in the air, in 3D space. Typically this was immediately confusing, with children asking 'how do you put it on?' Half way through the interaction with the table, one child picked up the torch to shine onto another object, but the light beam disappeared. In another group, also half way through interaction, a boy became very curious once he realized the torch was off: "how is it working if it's not even on?". This raises issues of expectation of use and mapping real-world objects to virtual concepts of interaction. A 'real' physical torch suggests use and practices that are appropriate in the real world, but not necessarily in the tangible world. The choice of a real torch was consistent with the use of real blocks that represented themselves - so that there was a consistent literal physical correspondence underlying the design of the environment. Although not difficult to use once explained, the intuitive action (because it is a real torch that is providing a digital output) to pick it up and move it around rather than leave it on the table highlight that constraints of the system do not always map to afforded or familiar interaction in the real world. This suggests that consistency of metaphor may not always be an appropriate design choice, and raises issues of trade-offs between employing a literal physical correspondence design in order to exploit familiar interaction and inferences; and the subsequent interactions that do not map well to the environment.

\section{Literal versus symbolic}

A further finding relating to literal versus symbolic physical correspondence emerged. With literal correspondence objects act 'as themselves', whereas with symbolic correspondence objects act as signifiers for or are representative of something else. In the post activity of our 
study students were required to use 3 clear objects that behaved as coloured ones, to establish whether they could determine which colour each block represented using the knowledge they had gained during interaction. However, several children were unable to transfer from perceiving objects with a literal correspondence to a symbolic correspondence, even though it was an independent activity, and relied on the same modality for testing.

F - I'd like you to tell me which colour you think these objects are...

P1 - White!

P2 - See-through!

F - But they're behaving as if they were of some other colour

So pupils try the objects on the table and one says: "that's weird!"

Thus, confusion occurs between reality and artificiality. Everything shown was artificially created for our purposes, but children at some point believe it's actually dependent on the real properties of the objects (e.g. it's reflecting green because the object really is green). So, when clear objects behaved as coloured ones, it introduced some confusion, as if the 'rule' had inexplicably changed ("how does it pick up a colour like that??", "why is it coming out green??"). Some pupils looked for other 'physical' explanations like "maybe the things inside it" referring to incidental air bubbles within the object. Eventually, the students could understand that it was the technical aspects that made a difference, but nevertheless this raises questions about the design of testing activities within the 'artificial' environment.

\section{CONCLUSION}

Few frameworks for conceptualising tangible environments in the context of learning have been developed. Taking into account the important role of external representations in mediating cognition $[24 ; 12 ; 1 ; 20 ; 4]$ and the complexity of the cognitive relationship with external representations in tangible environments, a representation-based framework for analyzing TUIs from the perspective of learning was developed [21]. Through studies using a purpose-built tangible environment, two parameters of this framework were analysed: location (co-located/ discrete) and physical correspondence (symbolic/ literal).

Collectively the findings suggest a number of implications for tangible environments for learning. Design findings, that different location of representation (discrete, colocated and embedded) lends itself to representing concepts at different of levels of abstraction. This is useful for considering design ideas particularly within scientific concepts, where understanding phenomena at different levels of abstraction is important. Findings that indicate interaction differences in the two location modes have some key implications for learning. Observed differences in group awareness suggest the value of explicit awareness of others action (in the co-located mode) in facilitating exploration, collaborative construction, and interpretation.
In addition, the rapid dynamic nature of the co-located approach was valuable in promoting increased access to exemplary instances of phenomena enhancing explorative activity. On the other hand slower interaction in the discrete mode allowed more 'time' for thinking. This raises questions about the value and realization of different forms of reflection - reflection in action and reflection on action for learning with co-located shared interfaces. This highlights the need to specifically design learning activities that slow down interaction and promote opportunities for reflection to occur during 'calm' periods at various points in the learning task.

In terms of physical correspondence, issues were raised around mappings of real-world objects to virtual, artificial environments, in which the object behaves as itself. Although the torch was actually representing a torch, it could not be turned on or used in the 3D space in the same way as in the real world. Thus, the system constraints on objects or actions do not necessarily map to familiar interaction in the real world. Furthermore, swapping between literal and symbolic mappings with the same kind of objects proved to be problematic, as children internalized couplings between physical properties of the objects and their behaviour in the digital world, and did not easily understand changes in those metaphors. This highlights issues around design of tangible interfaces and the potential impact on learning of mixed metaphors or requirements to shift from one metaphor to another.

\section{ACKNOWLEDGMENTS}

This research is supported by the EPSRC: EP/F018436. We also thank students and teachers from Woodlands and Sweyne Park schools for their participation in the research.

\section{REFERENCES}

1. Ainsworth, S., Bibby, P. \& Wood, D. (2002) Examining the effects of different multiple representational systems in learning primary mathematics. Journal of the Learning Sciences. 11, 1, 25-62.

2. Antle, A. (2007) The CTI framework: informing the design of tangible systems for children. Proceedings of the $1^{\text {st }}$ International Conference on Tangible and Embedded Interaction, ACM Press 195-202.

3. Fishkin, K. P. (2004). A taxonomy for and analysis of tangible interfaces. Personal and Ubiquitous Computing, 8 (5), 347-358

4. Garcia-Ruiz, M.A. (2001). Using non-speech sounds to convey molecular properties in a virtual environment International Conference of New Technologies in Science Education (CINTEC), Aveiro, Portugal, July.

5. Goldstone, R. L. and Son, J. Y. (2005) The Transfer of Scientific Principles Using Concrete and Idealized Simulations. The Journal of the Learning Sciences, 14 (1), 69-110.

6. Hornecker E., and Buur, J. (2006) Getting a Grip on Tangible Interaction: A Framework on Physical Space 
and Social Interaction. Proceedings of CHI 2006. Montreal. ACM Press, 437-446.

7. Hornecker, E., Marshall, P., Dalton, S. and Rogers, Y. (2008) Collaboration and Interference: Awareness with Mice or Touch Input. Proceedings of Computer Supported Collaborative Work. San Diego, USA.

8. Jorda, S. (2003) Sonigraphical Instruments: From FMOL to the reacTable. Proceedings of the 3rd Conference on New Interfaces for Musical Expression (NIME 03), Montreal (Canada).

9. Kaltenbrunner, M. and Bencina, R. (2007) reacTIVision: A Computer-Vision Framework for Table-Based Tangible Interaction. Proceedings of the first international conference on "Tangible and Embedded Interaction. Baton Rouge, Louisiana.

10.Klemmer, S., Hartmann, B. and Takayama, L. (2006) How Bodies Matter: Five Themes for Interaction Design. Designing Interactive Systems Penn State, USA

11. Koleva, B, Benford, S, Hui Ng, K. and Rodden, T. (2003) A Framework for Tangible User Interfaces. Physical Interaction Workshop on Real World User Interfaces. Mobile HCI, Udine, Italy.

12.Larkin, J. and Simon, H. (1987) Why a diagram is (sometimes) worth ten thousand words Cognitive Science 11, 69-100.

13. Lowe, R. (1999) Extracting information from an animation during complex visual learning. Eur. Journal of Psychology of Education Vol. XIV, 2, 225-244.

14. Marshall, P. (2007) Do tangible interfaces enhance learning? Proceedings of First International Conference on Tangible and Embedded Interaction, USA.

15. Marshall, P., Hornecker, E., Morris, R., Rogers, Y. and Dalton, N. (2008) When the fingers do the talking: A study of group participation for different kinds of shareable surfaces. Proceedings of IEEE Tabletops and Interactive Surfaces. Amsterdam October 2008.

16. O'Malley, C. and Fraser, D. S. (2004). Literature review in learning with tangible technologies. Technical Report 12, NESTA Futurelab.

17.Patten, J., Ishii, H., Hines, J., and Pangarp, G. (2001) Sensetable: a wireless object tracking platform for tangible user interfaces. In Proceedings of the SIGCHI Conference on Human Factors in Computing Systems (CHI'01), Seattle, USA. ACM Press.

18.Pinelle, D., Nacenta, M., Gutwin, C. and Stach, T. (2008) The effects of co-present embodiments on awareness and collaboration in tabletop groupware. International Proceedings of Graphics Interface Ontario, Canada.
19.Pontual Falcão, T., Price, S., Sheridan, J.G. Extending concepts of engagement in tangible environments. To appear in Workshop Notes of CHI 2009 [In press].

20.Price, S. (2002) Animated Diagrams: How effective are explicit dynamics for learners? In P. Bell, R. Stevens, \& T. Satwitz (eds), Keeping Learning Complex: Proceedings of the Fifth International Conference of the Learning Sciences 344-351. Mahwah, NJ: Erlbaum.

21.Price, S. (2008) A Representation Approach to Conceptualising Tangible Learning Environments. Proceedings of the Second International Conference on Tangible and Embedded Interaction, Bonn, Germany.

22.Price, S., Sheridan, J.G., Pontual-Falcao, T. and Roussos, G. (2008) Towards a Framework for Investigating Tangible Environments for Learning. Special Issue of International Journal of Arts and Technology on Tangible and Embedded Interaction.

23. Rogers, Y. and Lindley, S. (2004) Collaborating around vertical and horizontal displays: which way is best? Interacting With Computers, 16, 1133-1152

24. Scaife, M and Rogers, Y (1996) External Cognition: how do graphical representations work? International Journal of Human-Computer Studies 45, 185-213.

25. Scarlatos, L. L., Dushkina, Y., and Landy, S. (1999). TICLE: A tangible interface for collaborative learning environments. Extended Abstracts of the SIGCHI Conference on Human Factors in Computing Systems (CHI'99), pages 260-261, Pittsburgh, USA.

26. Scarlatos, T. and Scarlatos, L. (2000). Tangible Math Applications.

http://www.cs.sunysb.edu/ tony/TSresearch.htm. Accessed on: 31 October 2008

27. Stenning, K. (1998) Distinguishing Semantic from Processing Explanations of Usability of Representations: Applying Expressiveness Analysis to Animation. In (ed) J. Lee, Intelligence and Multimodality in Multimedia Interfaces: Research \& Applications AAAI Press.

28. Ullmer, B. and Ishii, H. (2001) Emerging frameworks for tangible user interfaces. Carroll, J. ed. Human Computer Interaction in the New Millenium, AllisonWesley, 2001, 579-601.

29. Underkoffler, J. \& Ishii, I. Illuminating Light: An Optical Design Tool with a Luminous-Tangible Interface. In Proceedings of the SIGCHI Conference on Human Factors in Computing Systems (CHI'98), Los Angeles, USA. ACM Press.

30.Zuckerman, O. Grotzer, T. \& Leahy, K. (2006) Flow blocks as a conceptual bridge between understanding the structure and behavior of a complex causal system. Proceedings of the 7th International Conference of the Learning Sciences Indiana, 880-886 